\title{
Development of Landscape Models for Conservation of Freshwater Mussels in the Upper Mississippi River Basin
}

Freshwater mussels are the most imperiled group of animals in North America. About $70 \%$ of the 300 native species are considered extinct, endangered, threatened, or of special concern. Freshwater mussels are a renewable resource, providing significant ecological and economic benefits to the Nation. Mussels serve as a food resource for many animals; they improve water quality by filtering contaminants, sediments, and nutrients; and their shells are used in producing cultured pearls. Their declines may be a signal of critical problems in river ecosystems.

Although many threats may be contributing to the declines in species density and diversity, past efforts have not determined which threats are most important. Because freshwater mussels are declining nationwide, it is important to understand what contributes to their distribution (location) and abundance (numbers) across large geographic areas. Much of the research on mussels has been conducted on small streams by teams of mussel specialists measuring traditional habitat variables. Most attempts to predict the distribution or abundance of mussels from variables such as substrate type or water depth have failed when tested critically, suggesting that these measures are inadequate descriptors of mussel communities. Also, many freshwater mussels require a fish host to complete their life cycle. Therefore, management approaches need to maintain the connection between mussels and their host fish. Further advances in understanding mussel populations will probably require a multidisciplinary approach that includes biology, chemistry, and hydrology in both large and small geographic areas.

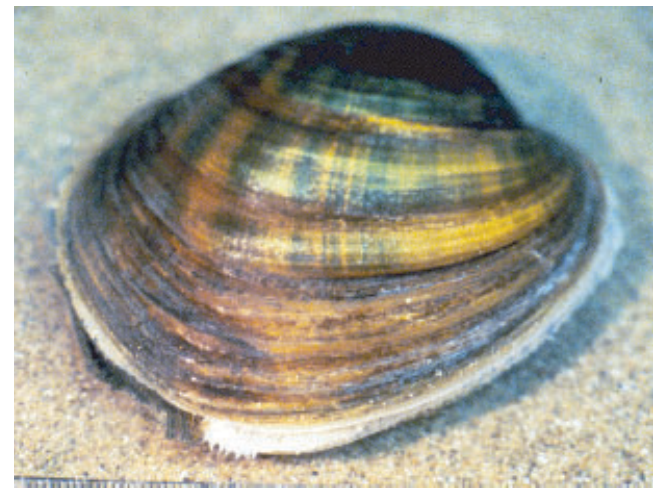

The Higgins eye (Lampsilis higginsi) is one of several freshwater mussel species in the Upper Mississippi River Basin on the Federal endangered species list.

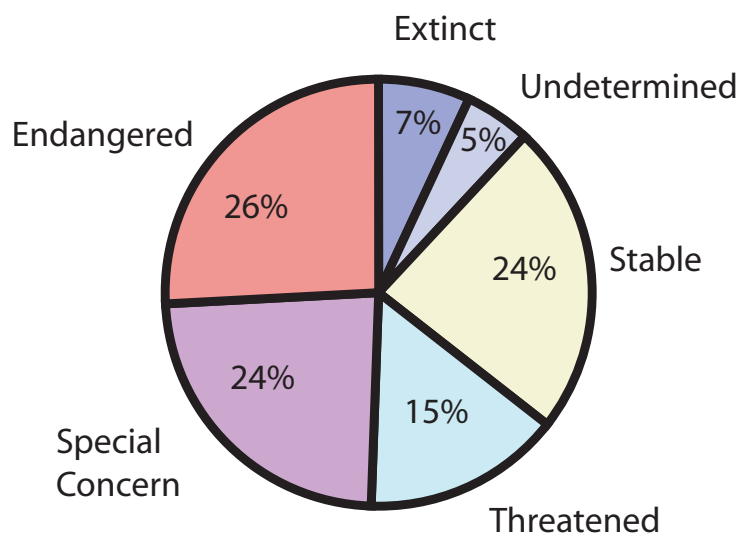

Declines in the number of species and abundance of freshwater mussels have been observed in many large rivers. The Nature Conservancy estimates that $72 \%$ of mussel species are vulnerable to extinction or are already extinct.

In 2001, scientists at the Upper Midwest Environmental Sciences Center began to develop predictive models. These computer models relate the distribution of freshwater mussels to physical and biological variables over large and small geographic areas. A geographic information system (GIS) allows scientists to examine the relations among many databases and provides graphical displays of these data. We developed a GIS database on the distribution and abundance of native mussels from eight studies conducted from 1975 to 2001. These studies were conducted in Navigation Pool 8, a 38-km reach of the Upper Mississippi River near La Crosse, Wisconsin. The data from the Pool 8 studies cover 587 locations and provide good geographic coverage throughout the pool, although most locations are near the deepest part of the channel. Of the 587 locations, roughly $60 \%$ contained mussels representing 37 species, including the endangered Higgins eye (Lampsilis higginsi). Densities of freshwater mussels were highly variable, ranging from 0 to 130 mussels per square meter.

We also assembled a database on physical and biological variables of Pool 8 to compare to mussel distributions. Physical features included land cover in the surrounding area; aquatic area type (e.g., main navigation channel, backwater lake); longitudinal position within the pool; proximity to wing dams, tributaries, and deep holes; and various hydraulic

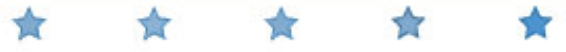


variables. We are also interested in determining if certain biological attributes influence mussel distribution. We grouped mussels into guilds on the basis of breeding strategy, conservation status, commercial status, shell shape, and the number of different fish families that serve as fish hosts. We are now investigating relations among the biological and physical variables and building simple models to generate hypotheses for future research.

Recent analysis suggests that several hydraulic variables may be important in predicting the distribution of mussels. For example, mussels are most often found in areas with a combination of low shear stress (the force exerted by moving water near the river bottom) during periods of high flows and some minimum level of current velocity during low flow periods. Such areas may allow mussels to maintain their position during floods (low shear stress) and still provide adequate food resources, oxygen, and removal of waste products during low water periods. Preliminary analysis shows that three variables-velocity, depth, and shear stress-can predict the presence of mussels $80-96 \%$ of the time. Of the 587 locations, however, only about 50 meet the optimal conditions predicted by these models. We don't know whether these conditions are found in unsampled locations or only in a very small percentage of the pool.

Certain biological attributes may also influence the distribution of mussels. For example, thin-shelled species were most common in the upper portion of the pool. The upper portion is more river-like than the lower dammed portion, which is more lake-like. In contrast, those locations dominated by thick-shelled species were evenly distributed throughout the pool. Mussels in the subfamily Anodontinae (short-lived, early maturing, rapid growth) were predominately found in the main navigation channel. Mussels in the subfamilies Lampsilinae (long-lived, late maturing, slow growth) and Unioninae (moderate life span, moderate maturity, and moderate growth) were more widely distributed among other aquatic areas in the pool.

Present analysis focuses on dividing the 587 locations into those with and without juveniles and those with low and high mussel densities. Initially, we will use classification and regression techniques to categorize mussels into groups with similar physical and biological characteristics. We will then develop more detailed analytical models using those variables that seem to influence the distribution of mussels.

We conducted the study using Navigation Pool 8 because the USGS-administered Long Term Resource Monitoring Program provides 15 years of data about physical features (velocity models, depths) and biological components (fish, invertebrates, plants). However, this type of analysis could be done in other data-rich locations at large and small geographic scales and should be broadly applicable throughout other areas such as the Great Lakes region.

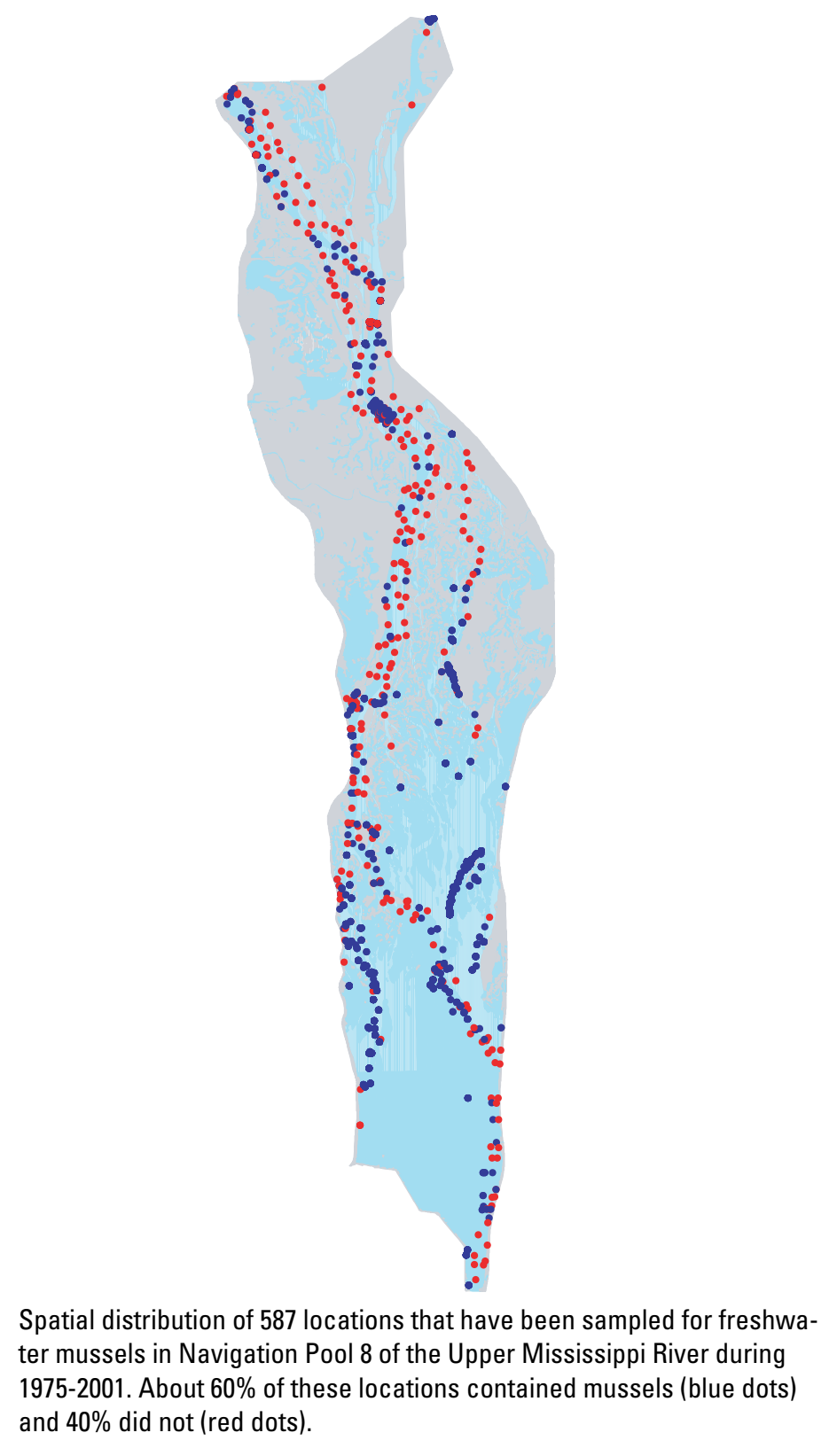

See the project Web page for more information. http: //www.umesc.er.usgs.gov/aquatic/native_mussels/ 5004455.html

\section{For more information, contact Teresa Newton}

Upper Midwest Environmental Sciences Center 2630 Fanta Reed Road,

La Crosse, WI 54603

Phone: 608.783 .6217

Email: tnewton@usgs.gov

http://www.umesc.usgs.gov/ 